18

\title{
Об интерпретации спектров фотохимически индуцированного кругового дихроизма полупроводниковых нанокристаллов
}

\author{
(C) Ф.М. Сафиин, В.Г. Маслов \\ Университет ИТМО, \\ 197101 Санкт-Петербург, Россия \\ e-mail: farruhsafin@gmail.com
}

Поступила в редакцию 03.02.2020 г.

В окончательной редакции 03.02.2020 г.

Принята к публикации 28.02.2020 г.

\begin{abstract}
Предложен подход к анализу и интерпретации спектров фотоиндуцированного кругового дихроизма (ФИКД) полупроводниковых нанокристаллов, в частности, квантовых точек, основанный на их сопоставлении со спектрами магнитного кругового дихроизма (МКД). Показано, что спектры МКД и ФИКД имеют схожие особенности. По спектрам ФИКД определены величины параметров, аналогичные известным параметрам $A$ и $B$ спектров МКД, характеризующих расщепление и перемешивание аналогичных состояний.
\end{abstract}

Ключевые слова: фотоиндуцированный круговой дихроизм, оптическая активность, хиральность, квантовые точки, магнитный круговой дихроизм, квантовые нанокристаллы.

DOI: $10.21883 /$ OS.2020.06.49402.57-20

\section{Введение}

Хиральность - одно из фундаментальных свойств физического мира. Хиральные нанокристаллы могут оказать существенное влияние на нанотоксикологию и нано-биотехнологии [1-6]. Потенциально нанокристаллы, кристаллическая решетка которых обладает относительно высокой степенью симметрии, могут быть хиральными из-за наличия дефектов на их поверхности или в их объеме [7-9]. В целом ансамбль нанокристаллов, содержащий такие дефектные нанокристаллы, не обладает оптической активностью. Это объясняется наличием в смеси равного количества левовращающих и правовращающих энантиомеров, а также, возможно, некоторого количества частиц, не имеющих дефектов, то есть не обладающих хиральностью (оптически неактивных). Развитие данной концепции привело к идее разделения энантиомеров нанокристаллов посредством энантиоселективного межфазового перевода [7].

Ранее мы сообщали об исследовании оптической активности, которая была вызвана фотохимической реакцией при освещении квантовых точек (КТ) циркулярно поляризованным светом. Фотоиндуцированный круговой дихроизм был оценен количественно, и было показано, что фотоиндуцированная химическая реакция протекает избирательно, в зависимости от направленности циркулярно поляризованного света [10].

Целью данной работы является разработка подходов к анализу и интерпретации спектров кругового дихроизма (КД), полученных в экспериментах по исследованию фотохимически индуцированного кругового дихроизма (ФИКД) полупроводниковых квантовых нанокристаллов, в частности, квантовых точек $\mathrm{CdSe} / \mathrm{ZnS}$.

\section{Результаты и обсуждение}

Предполагаем, что исследуемый образец содержит равные количества обоих энантиомеров поглощающих свет центров. Будем также предполагать, что в результате фотохимической реакции под действием циркулярного поляризованного света происходит фотообесцвечивание центров. В этом случае изменение оптической плотности определяется выражением: $\delta D(\lambda)=-\varepsilon(\lambda) z \delta c$, где $\varepsilon-$ коэффициент экстинкции, $z$ - длина пути, $\delta c-$ изменение концентрации в результате фотохимической реакции. При этом спектр ФИКД совпадает (с точностью до множителя, постоянного по спектру) со спектром КД одного из энантиомеров (того, который больше поглощает на длине волны облучения) со знаком минус. Можно записать:

$$
\begin{aligned}
\delta D(\lambda) & \sim-D(\lambda), \\
\Delta D_{P I C D}(\lambda) & \sim-\Delta D_{1,2}(\lambda),
\end{aligned}
$$

где $\delta D(\lambda)$ - разностный спектр поглощения (спектр после реакции минус спектр до реакции $), D(\lambda)$ - спектр поглощения исходного вещества, $\triangle D_{P C D}-$ спектр ФИКД (спектр КД после фотореакции минус спектр КД до реакции), $\Delta D_{1,2}$ - спектр кругового дихроизма (КД) чистых энантиомеров. Здесь для определенности предполагается, что облучение при проведении фотореакции осуществляется $L$-поляризованным светом, а спектры КД измеряются согласно определению: $\Delta D=D_{L}-D_{R}$. Случай облучения $R$-поляризованным светом может быть рассмотрен аналогичным образом.

Предполагается, что КД отдельных центров вызван статическим асимметричным возмущением, которое достаточно для возникновения оптической активности, в 
частности, не имеет центра инверсии и плоскостей симметрии. Поскольку формальные причины возникновения КД в данном случае аналогичны тем, которые имеют место в случае магнитного кругового дихроизма (МКД): расщепление вырожденных состояний (члены $A$ и $C$ ) и вызванное возмущением перемешивание состояний (член $B$ ), то в принципе спектр КД, наведенного таким возмушением, может описываться теми же соотношениями, что и спектр МКД [11]:

$$
\begin{aligned}
\frac{\Delta D_{M C D}(E)}{E}= & \gamma\left\{A_{1}\left(-\frac{d f(E)}{d E}\right)\right. \\
& \left.+\left(B_{0}+\frac{C_{0}}{k T}\right) f(E)\right\}\left(\beta_{B} H\right) c z,
\end{aligned}
$$

где $\Delta D_{M C D}(E)$ - спектр МКД, $E=h v, A_{1}, B_{0}, C_{0}-$ параметры, определяющие вклады членов $A, B, C$ (при комнатной температуре обычно полагают $C_{0}=0$ ), $f(E)$ - нормированная кривая поглощения в области исследуемого перехода $\int_{0}^{\infty} f(E) d E=1, k-$ постоянная Больцмана, $T$ - температура, $\beta_{B}-$ магнетон Бора, $H$ - напряженность магнитного поля, $\gamma-$ константа, пропорциональная силе осциллятора перехода.

Формула, описывающая спектр поглощения $D_{L, R}^{0}(E)$ в отсутствии магнитного поля, в данных обозначениях имеет вид:

$$
\frac{D_{L, R}^{0}(E)}{E}=\gamma D_{0} f(E) c z,
$$

где $D_{0}$ - характеристика перехода, равная квадрату дипольного момента перехода (в $\left.D^{2}\right)$.

Для характеристики численных значений $A_{1}, B_{0}$ обычно используют относительные величины $A_{1} / D_{0}$ и $B_{0} / D_{0}$. Для определения их из экспериментального спектра используют формулы [13]:

$$
\begin{gathered}
\frac{A_{1}}{D_{0}}=\frac{e^{1 / 2}}{2 \times 2.35 \beta_{B}} \frac{(D i f) \Gamma}{D_{m} H}, \\
\frac{B_{0}}{D_{0}}=\frac{1}{\beta_{B}} \frac{\Delta D_{m}}{D_{m} H}
\end{gathered}
$$

где $\Gamma$ - полуширина полосы поглощения в $\mathrm{cm}^{-1}$, $D_{m}$ - значение оптической плотности в максимуме поглощения в рассматриваемом переходе, $\Delta D_{m}-$ значение МКД в максимуме (или минимуме) поглощения в данном переходе, $($ Dif ) - разница между максимумом (минимумом) на длинноволновом спаде спектра МКД в области данного перехода и минимумом (максимумом) на его коротковолновом спаде, $H-$ напряженность магнитного поля, $1 / \beta_{B}=2.141 \mathrm{~T} / \mathrm{cm}^{-1}$.

В случае статического хирального возмущения вместо $\beta_{B} H$ должна фигурировать величина, характеризующая возмущение, но вместо этого мы можем переопределить $A_{1}$ и $B_{0}$. Вместо $A_{1}$ и $B_{0}$ будем использовать величины $a_{1}$ и $b_{0}$, характеризующие каждый электронный переход с точки зрения его „чувствительности“ к действию возмущения, при этом величина $a_{1}$ характеризует расщепление вырожденных состояний и величина $b_{0}$ характеризует смешивание состояний. В этом случае спектр ФИКД может быть выражен формулой:

$$
\begin{aligned}
\frac{\Delta D_{P C D}(E)}{E}= & \gamma\left\{a_{1}\left(-\frac{d f(E)}{d E}\right)\right. \\
& \left.+\left(b_{0}+\frac{c_{0}}{k T}\right) f(E)\right\} c z .
\end{aligned}
$$

Соотношения для $a_{1}$ и $b_{0}$, аналогичные формулам (5), (6), в этом случае примут вид:

$$
\begin{gathered}
\frac{a_{1}}{D_{0}}=\frac{e^{1 / 2}}{2 \times 2.35} \frac{(\text { Dif }) \Gamma}{D_{m}}, \\
\frac{b_{0}}{D_{0}}=\frac{\Delta D_{m}}{D_{m}} .
\end{gathered}
$$

Величина $a_{1} / D_{0}$ имеет размерность $\mathrm{cm}^{-1}$ и связана с величиной расщепления $(\Delta E)$ вырожденных состояний. В случае МКД имеем (см., например, [11]): $\Delta E_{M C D}=2 \frac{A_{1}}{D_{0}} \beta_{B} H=\frac{e^{1 / 2}}{2.35} \frac{(D i f) \Gamma}{D_{m}}$. Аналогично в случае ФИКД легко получить: $\triangle E_{P C D}=2 \frac{a_{1}}{D_{0}}$.

В спектрах нанокристаллов различных типов, обычно бывают хорошо видны несколько полос поглощения, каждой из которых соответствует группа (мультиплет) переходов. По данным ФИКД мы можем получить „эффективные“ величины $a_{1}$ и $b_{0}$, имеющие смысл суммарных величин для всех переходов, входящих в данную группу.

Таким образом, можно ожидать определенной аналогии между спектрами МКД и ФИКД, т.е. совпадения положения основных особенностей, связанных с проявлениями членов $A$ и $B$. Однако соотношения между этими вкладами для разных переходов и даже знаки этих членов в спектрах МКД и ФИКД могут различаться.

Мы анализируем спектры ФИКД, которые были получены после 40-минутного воздействия циркулярно поляризованного света на раствор КТ и описаны в работе [10]. Для расчетов параметров $a$ и $b$ удобнее воспользоваться разностным спектром ФИКД (кривая 4 на рисунке). Спектры МКД измерялись в магнитном поле напряженностью $\pm 1.5 \mathrm{~T}$ на спектрофотометре кругового дихроизма Јasco-1500, оборудованном магнитным блоком MCD-581.

В спектре поглощения (кривая 1) отчетливо видны 2 полосы с максимумами на длинах волны $543 \mathrm{~nm}$ и $447 \mathrm{~nm}$. В спектре МКД (при $H=1.5 \mathrm{~T}$ ) длинноволновой полосе поглощения соответствуют особенности в виде максимума на $528 \mathrm{~nm}$ и минимума у $557 \mathrm{~nm}$, явно свидетельствующие о наличии вклада члена $A$ при незначительном вкладе члена $B$. Полосе поглощения $447 \mathrm{~nm}$ соответствуют максимум у $445 \mathrm{~nm}$ и минимум у $465 \mathrm{~nm}$, что указывает на вклад члена $A$ при возможном наличии также вклада $B$. Кроме того, в спектре МКД имеется широкий минимум в области $465-500 \mathrm{~nm}$, свидетельствующий о наличии между указанными полосами 
Параметры КД: $A, B, a, b$, энергия перехода, полуширина - для двух групп переходов

\begin{tabular}{c|c|c|c|c|c}
\hline Переход (максимум, nm) & $A_{1} / D_{0}$ & $B_{1} / D_{0}, \mathrm{~cm}$ & $a_{1} / D_{0}, \mathrm{~cm}^{-1}$ & $b_{0} / D_{0}$ & $\Gamma(F W H D)$ \\
\hline $1(543 \mathrm{~nm})$ & 0.6807 & $\leq 0.0009$ & 0.0038 & $3.5 \cdot 10^{-6}$ & $1344 \mathrm{~cm}^{-1}$ \\
$2(447 \mathrm{~nm})$ & 0.1432 & 0.0003 & $\geq 0.0001$ & $6 \cdot 10^{-6}$ & $1524 \mathrm{~cm}^{-1}$
\end{tabular}

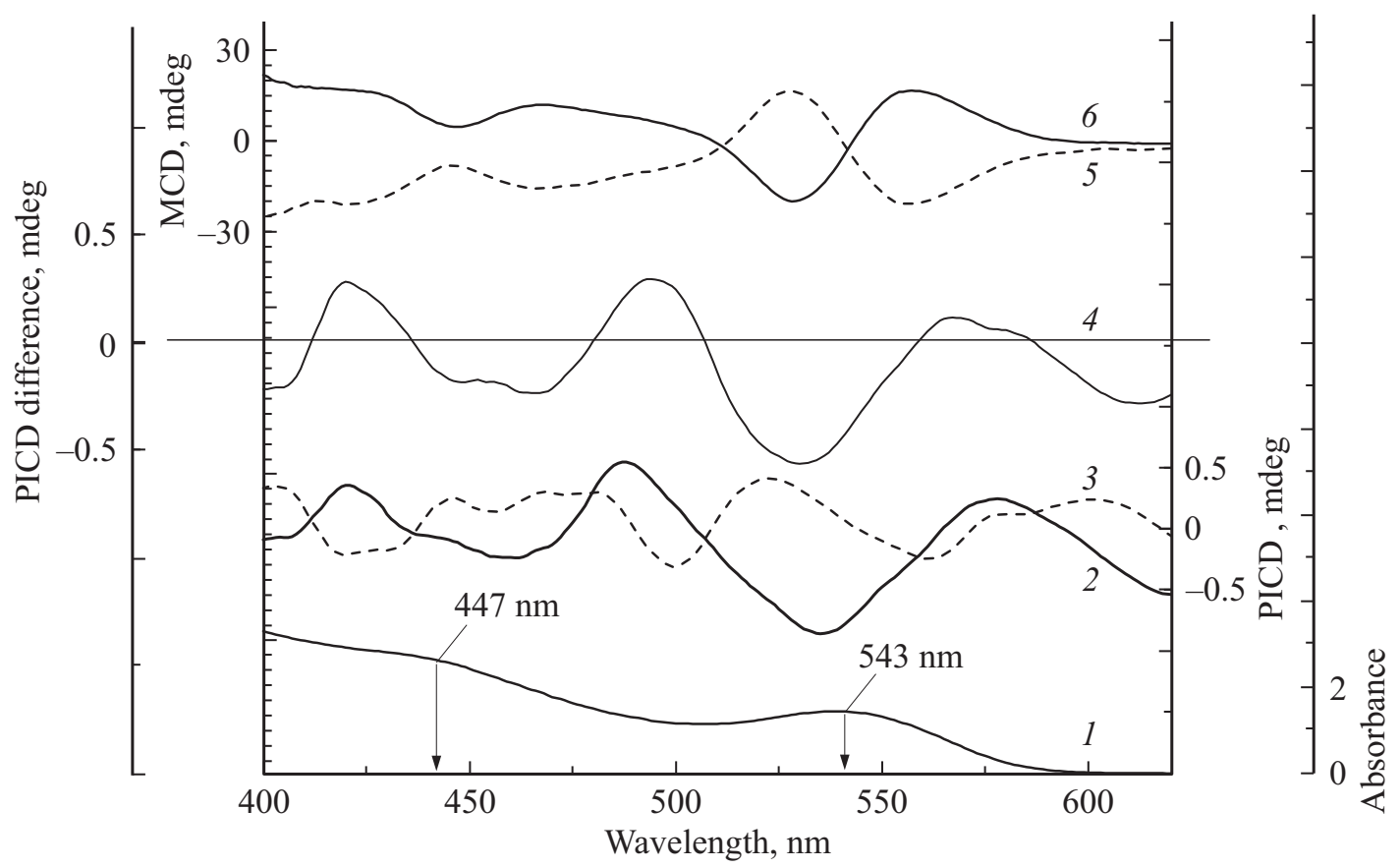

Спектры МКД и спектры ФИКД квантовых точек CdSe/ZnS в хлороформе: 1 - спектр поглощения; 2 - спектр ФИКД, полученный после 40 минут облучения светом с левой круговой поляризацией; 3 - спектр ФИКД, полученный после 40 mіn облучения светом с правой круговой поляризацией; 4 - разностный спектр ФИКД (ФИКД, полученный после 40 минут облучения светом с левой круговой поляризацией, минус ФИКД, полученный после 40 минут облучения светом с правой круговой поляризацией); 5 - спектр МКД при $H=+1.5 \mathrm{~T} ; 6-$ спектр МКД при $H=-1.5 \mathrm{~T}$.

поглощения еще одной группы переходов, не разрешенных в спектре поглощения, строгий анализ которой пока затруднителен. В том, что касается длинноволновой области, спектр МКД качественно согласуется со спектрами, описанными ранее в литературе для CdSe и $\mathrm{AgInS}_{2} / \mathrm{ZnS}$ KT $[12,14]$.

Если сравнить спектр ФИКД со спектром МКД, то обращает на себя внимание почти полная аналогия между ними. Почти каждой особенности спектра ФИКД соответствует та или иная особенность в спектре МКД, хотя имеются различия в положении и ширинах некоторых минимумов и максимумов. Так, длинноволновой полосе поглощения соответствуют в спектре ФИКД максимум $565 \mathrm{~nm}$ и минимум $530 \mathrm{~nm}$, что свидетельствует о наличии вклада члена $a$ при ненулевом (в отличие от спектра МКД) вкладе $b$. В области полосы $447 \mathrm{~nm}$ имеем минимум 446 и максимум $460 \mathrm{~nm}$, что также указывает на возможное наличие члена $a$. Однако спектр ФИКД в этой области сильно искажен влиянием соседних переходов, более сильным, чем в спектрах МКД.
В таблице приведены значения членов $A$ и $B$ в двух группах переходов, рассчитанные по формулам (5), (6) и (8), (9) из описанных выше спектров МКД их аналогов в спектрах ФИКД. Наличие члена $A$ указывает на вырожденность переходов, в то время как величина члена $B$ относительно мала. Подробное изучение МКД квантовых точек CdSe проводилось в работе [12], в которых также присутствует член $A$. В работе [13], посвященной изучению МКД нанопластин $\mathrm{CdSe} / \mathrm{CdS}$, говорится о наличии члена $B$, появление которого связывалось с двухмерной структурой нанопластин, в то время как для квантовых точек сферической формы характерно наличие лишь члена $A$.

Полученное значение параметра $a_{1} / D_{0}$ в длинноволновой полосе поглощения свидетельствует о том, что величина расщепления в данном переходе составляет $0.0076 \mathrm{~cm}^{-1}$ и более чем на два порядка меньше, чем величина зеемановского расщепления в МКД для этого же перехода при напряженности магнитного поля в $1.5 \mathrm{~T}$. 


\section{Заключение}

В работе показано, что спектры МКД и ФИКД имеют схожие особенности. Был предложен подход к анализу и интерпретации спектров ФИКД квантовых нанокристаллов, основанный на их сопоставлении со спектрами МКД, и продемонстрированы возможности этого подхода на примере квантовых точек $\mathrm{CdSe} / \mathrm{ZnS}$. Определены величины параметров, аналогичные известным параметрам $A$ и $B$ спектров МКД, характеризующих расщепление и перемешивание состояний. Получены численные значения соответствующих параметров.

\section{Финансирование работы}

Работа выполнена при поддержке Министерства науки и высшего образования Российской Федерации, госзадание № 2019-1080, и при финансовой поддержке Правительства Российской Федерации, грант 08-08.

\section{Конфликт интересов}

Авторы заявляют, что у них нет конфликта интересов.

\section{Список литературы}

[1] McKendry R., Theoclitou M.-E., Rayment T., Abell C. // Nature. 1998. V. 391. P. 566-568.

[2] Tamura M., Fujihara H. // J. American Chem. Soc. 2003. V. 125. P. $15742-15743$.

[3] Ben Moshe A., Szwarcman D., Markovich G. // ACS Nano. 2011. V. 5. P. $9034-9043$.

[4] Ma W., Xu L., de Moura A.F., Wu X., Kuang H., Xu C., Kotov N.A. // Chemical Reviews. 2017. V. 117. P. 8041-8093.

[5] Ben-Moshe A., Maoz B.M., Govorov A.O., Markovich G. // Chemical Society Reviews. 2013. V. 42. P. 7028-7041.

[6] Govorov A.O., Gun'ko Y.K., Slocik J.M., Gerard V.A., Fan Z., Naik R.R. // J. Materials Chem. 2011. V. 21. P. 16806-16818.

[7] Mukhina M.V., Maslov V.G., Baranov A.V., Fedorov A.V., Orlova A.O., Purcell-Milton F., Govan J., Gun'ko Y.K. // Nano Lett. 2015. V. 15. P. 2844-2851.

[8] Govorov A.O., Gun'ko Y.K., Slocik J.M., Gérard V.A., Fan Z., Naik R.R. // J. Materials Chem. 2011. V. 21. P. 16806-16818.

[9] Baimuratov A.S., Pereziabova T.P., Zhu W., Leonov M.Y., Baranov A.V., Fedorov A.V., Rukhlenko I.D. // Nano Lett. 2017. V. 17. P. 5514-5520.

[10] Safin F., Kolesova E., Maslov V., Gun'ko Y., Baranov A., Fedorov A. // J. Phys. Chem. C. 2019. V. 123. P. 19979-19983.

[11] Kobayashi N., Muranaka A. Circular dichroism and magnetic circular dichroism spectroscopy for organic chemists. Royal Society of Chemistry, 2011, P. 41.

[12] Kuno M., Nirmal M., Bawendi M.G., Efros A., Rosen M. // J. Chem. Phys. 1998. V. 108. P. 4242-4247.

[13] Gromova Y.A., Miropoltsev M.A., Cherevkov S.A., Maslov V.G., Baranov A.V., Fedorov A.V. // Opt. Spectrosc. 2018. V. 125 . P. $698-702$.

[14] Gromova Y., Sokolova A., Kurshanov D., Korsakov I., Osipova V., Cherevkov S., Baranov A. // Materials. 2019. V. 12. P. 3616. P. 1-12. 